\title{
A High Performance EO Small Satellite Platform \& Optical Sensor Suite
}

\author{
Mike Cutter, Philip Davies, Adam Baker, Martin Sweeting \\ Surrey Satellite Technology Limited, SSTL \\ Guildford, United Kingdom \\ $\underline{\text { m.cutter@sstl.co.uk, a.baker@sstl.co.uk }}$
}

\begin{abstract}
This paper describes a new high performance cost effective Earth Observation Platform, the SSTL-300 and associated optical sensor suite, offering a 7-year mission lifetime with a very high operational availability, for a mission cost an order of magnitude less than commercial high resolution Earth observation spacecraft. The paper will detail the SSTL-300 main payload, a very high-resolution imager with panchromatic multispectral channels. The paper will also outline an additional payload, the Medium Resolution Imager (MRI), which offers the widest swath of any comparable Earth Observation spacecraft. Earth images can be geolocated with high accuracy without the need for ground control points. Simultaneous high- and mediumresolution imaging is possible, coupled with either on-board lossless data compression for store-and-forward operations or near real-time imaging \& down-linking for a range of targets close to the ground station.
\end{abstract}

The SSTL-300 offers a range of imaging modes, including: strip mapping, fast response scene capture and stereo imaging offering swath widths up to $60 \mathrm{~km}$ at high-resolution. Details of the nominal (sun-synchronous) orbit for the SSTL-300 will be provided stressing the balance between single spacecraft and constellation (multiple cooperating spacecraft) performance. The latter requires an accurate and reliable, yet low cost propulsion system which SSTL has developed, but is commonly featured on low cost small spacecraft.

Keywords - small satellite, optical sensor, Earth observation, cost-effective, constellation, DMC

\section{SSTL'S HERITAGE IN EARTH OBSERVATION}

The first five satellites of the Disaster Monitoring Constellation (DMC) launched in 2002-2005 demonstrated the humanitarian, political and technological value of Earth imaging using cost-effective small satellites. Having met the primary objectives of their buyers, DMC spacecraft are facilitating the delivery of commercial earth observation data services. The British National Space Centre provided $£ 15 \mathrm{M}$ in funding through the MOSAIC (MicroSatellite Applications in Collaboration) program from 2000 which helped develop the SSTL 'enhanced' microsatellite platform and camera used for the DMC spacecraft.

MOSAIC has now leveraged significant export sales, commencing with AlSat-1 for the Algerian Space Agency (CNTS). The UK government, the Nigerian Agency for Space Research \& Development (NASRDA), the Turkish Ministry of
Science and Technology research institute BILTEN TUBITEK and the Chinese Ministry of Science \& Technology have since purchased Disaster Monitoring Constellation spacecraft.

The partners listed above formed the DMC consortium, agreeing to reserve 5\% of system capacity for disaster management, to operate as part of a constellation, to share data, and to investigate commercial exploitation of the data. In November 2005, the DMC, coordinated by SSTL's business unit DMC Imaging International (DMCii) officially joined the "International Charter: Space and Major Disasters", supplying broad daily image coverage during disasters such as hurricane Katrina and the Indian Ocean Tsunami.

The Beijing-1 microsatellite, built for the Beijing LandView Mapping Information Technology Co. (BLMIT), of Beijing, China, was added to the DMC constellation in October 2005. Beijing-1 is the first DMC+4 spacecraft built by SSTL. $\mathrm{DMC}+4$ combines the basic DMC wide area multispectral payload, operating at $32 \mathrm{~m}$ Ground Sample Distance (GSD) at nadir and detailed in Section IV, with a 4m GSD panchromatic instrument, also detailed in Section IV. This pair of instruments will provide compatibility with DMC data, for example generating a cloud free map of China every 6 months, and enable out systematic mapping at a higher resolution in support of the 2008 Beijing Olympic Games. Beijing-1, which had a mass of $160 \mathrm{~kg}$ at launch, is classified as an SSTL-150 spacecraft and is shown undergoing final assembly, integration and test in Figure 1:

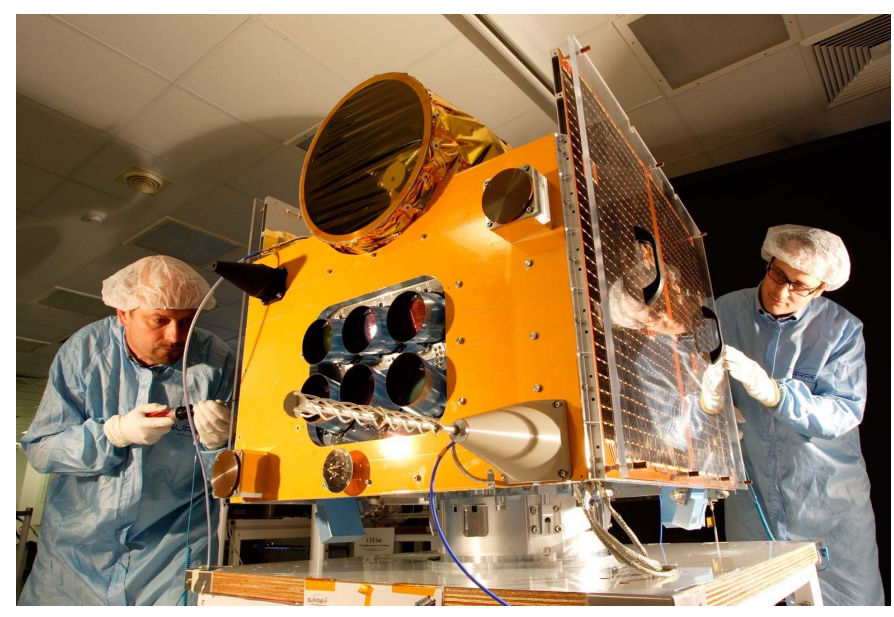

Figure 1. Beijing-1 microsatellite nearing assembly completion 
An example in-orbit high resolution image from Beijing-1 is shown in the Figure 2, taken over Valencia in August 2006:

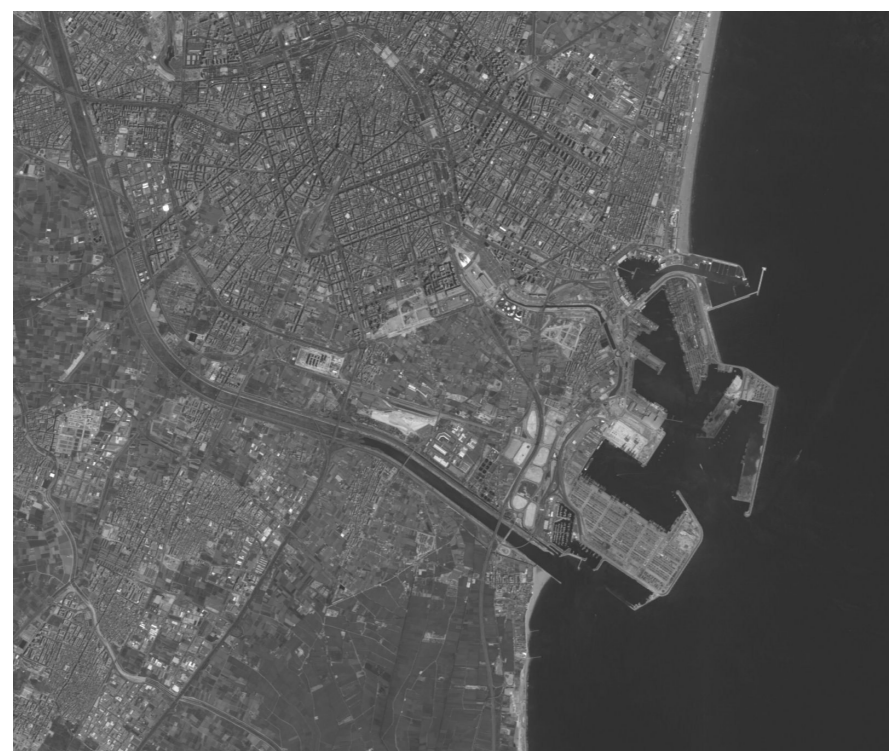

Figure 2. In-orbit image from Beijing-1, Valencia, Spain at $\sim 4 \mathrm{~m} \mathrm{GSD}$

The following image illustrates the potential of the wide area multispectral imager, which can deliver images up to $600 \mathrm{~km}$ wide and $2000 \mathrm{~km}$ long, limited only by the capacity of on-board data recorders and downlink data rates.

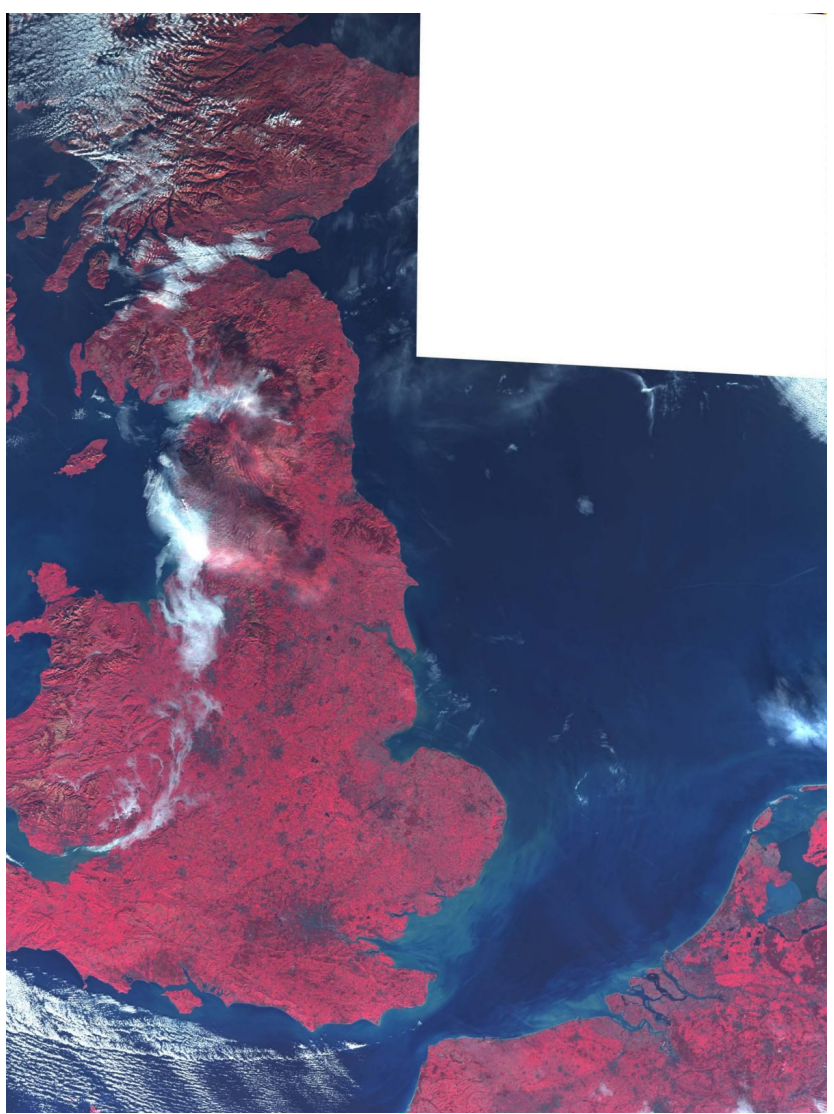

Figure 3. In-orbit image from Beijing-1, United Kingdom and NW France at $\sim 32 \mathrm{~m}$ GSD, colours Red, Green and Near Infra-red

\section{COST EFFECTIVE EARTH OBSERVATION FROM SPACE}

The key benefit of using small, cost effective spacecraft such as Bejing-1, delivered for a contract value of $\$ 18 \mathrm{M}$ including launch, ground station and early orbit operations is each small spacecraft can now offer capability that has previously only been available at much higher cost and on larger platforms. In constellations, i.e. when several spacecraft operate in collaboration, such as through flying at regularly spaced intervals in a single orbit plane, the price-performance balance is unmatched.

Specifically, constellations of small spacecraft enable high temporal resolution (i.e. frequent revisits over targets of interest) to be matched with high spatial resolution (GSD), for a total mission cost well within that of a single conventional large remote sensing spacecraft. Although spacecraft cannot approach the best resolutions offered by aerial platforms, the latest generation of high resolution commercial imaging spacecraft are claiming $0.5 \mathrm{~m}$ GSD at nadir [1]. However the revisit period of such spacecraft is typically 2-3 days, the best resolution is only gained in the image centre, and capability itself has cost in the order of $\$ 500 \mathrm{M}$ to develop (based on the published US government NextView contract value). In contrast, a typical small spacecraft such as NigeriaSat-2 can be built for an order of magnitude less, will offer a GSD as good as $2 \mathrm{~m}$, and can offer daily repeat with as few as 2 spacecraft, both of which can be launched using a single low cost rocket.

Figures 4 and 5 illustrate how constellations of small, low cost spacecraft can offer the unique blend of resolution, timeliness and utility for the maximum number of applications:

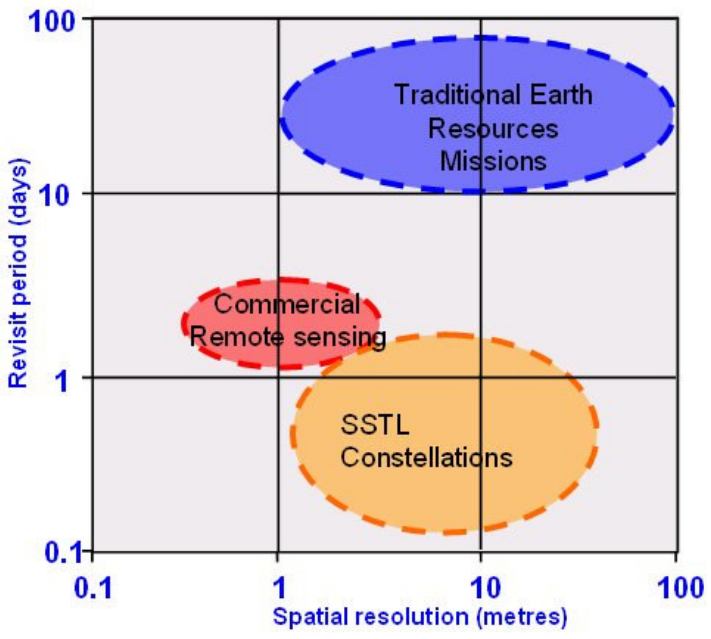

Figure 4. Comparison of revisit time v. resolution for traditional v. commercial high resolution v. SSTL spacecraft

The price-performance balance which a constellation of small satellites offers, coupled with its tolerance to failure, ability to be upgraded over time and low cost of entry (crucial to developing nations or small national programs) suggests that the future of Earth observation lies this way. 


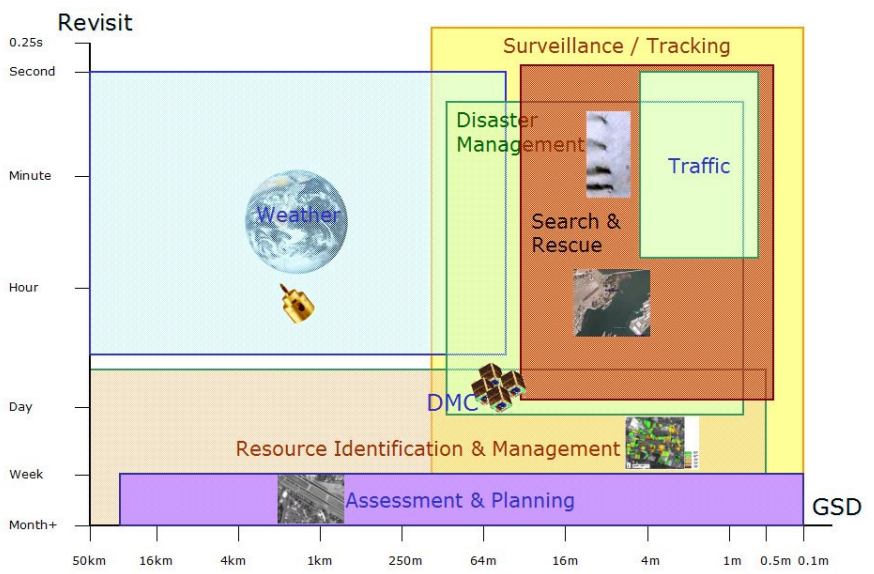

Figure 5. Comparison of revisit time v. resolution expressed in terms of application utility. Constellations such as DMC sit at the 'sweet spot' of maximum application overlap.

\section{High PERFORMANCE LOW COST EARTH OBSERVATION PLATFORM: THE SSTL-300}

The SSTL-300 and associated optical sensor suite has been developed to provide customers with a capability that has previously only been available at much higher cost and on larger platforms. This platform offers a 7-year mission lifetime with a very high operational availability.

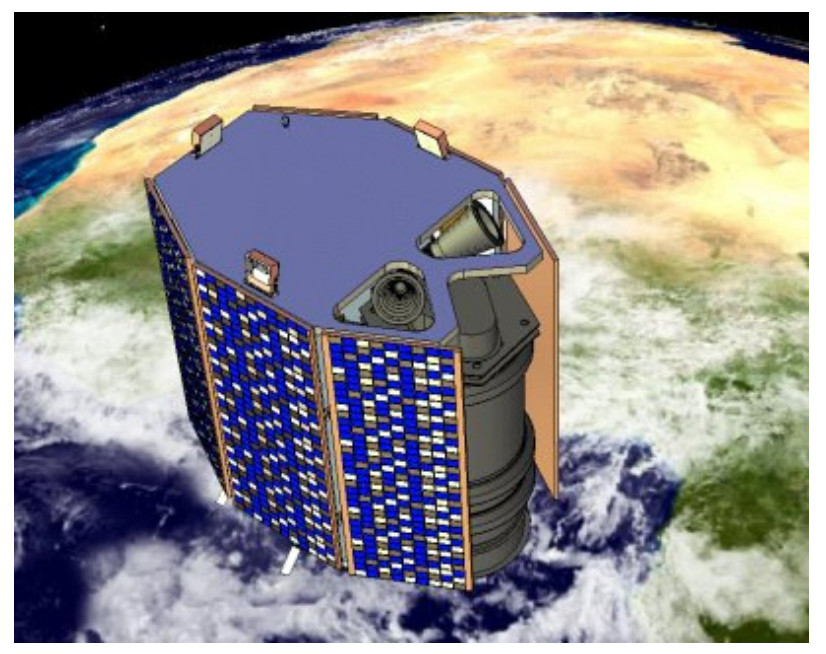

Figure 6. NigeriaSat-2, the first SSTL-300 spacecraft

The first SSTL-300, Nigeriasat-2 will provide Nigeria with valuable geographically referenced imaging for mapping, climate change monitoring, water resources management, agricultural planning, population estimation, health hazard monitoring and disaster mitigation and management.

Through Nigeriasat-2, Nigeria will continue contributing to the DMC, fully controlling the satellite from NASRDA's new satellite mission control centre facilities in Abuja, Nigeria.

Nigeriasat-2, weighing about 300 kilograms at launch, will feature a high-capacity solid-state onboard recorder with a 16gigabyte memory and a downlink capacity of up to 210 megabits per second in X-band.
Nigeriasat-2 will be able to produce images with a 2.5 meter ground resolution in black-and-white mode, and a 5meter resolution in multispectral mode. Designed to operate from a 700-kilometer orbit for seven years, the satellite will produce up to 400 images per day.

\section{SSTL EARTH OBSERVATION PAYLOADS}

\section{A. Very high high resolution cameras}

The SSTL-300's main payload is a very high-resolution imager (VHRI) with a panchromatic $2.5 \mathrm{~m}$ ground sampling distance (GSD) channel and four multispectral channels offering $5 \mathrm{~m}$ GSD.

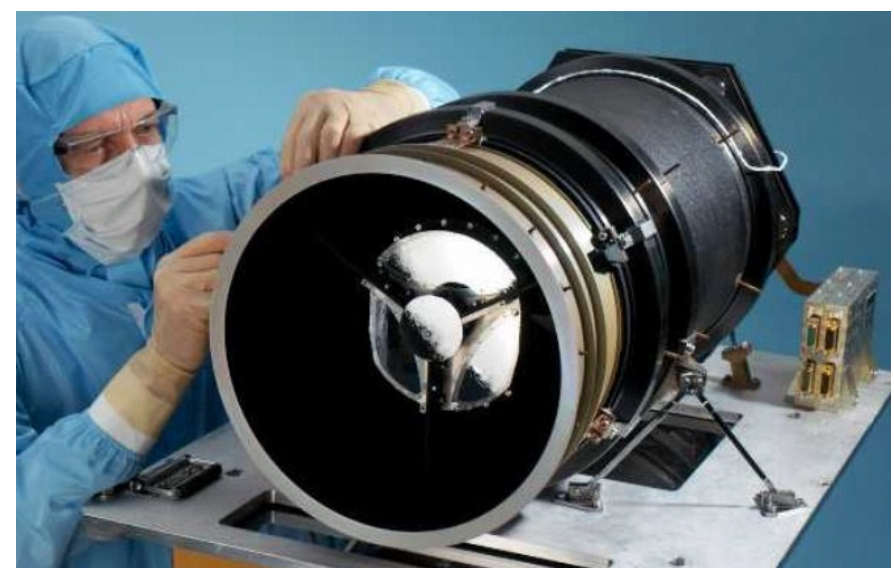

Figure 7. 4m GSD panchromatic and multispectral imager for Beijing-1 (2.5m GSD NigeriaSat-2 imager similar design)

The imager swath is $20 \mathrm{~km}$ in all channels. This imager is an extension of the 4m GSD imager already flying on Beijing-1, which was launched in October 2005.

\section{B. Wide area multispectral cameras}

Additional payloads can be accommodated such as the SLIM6 medium resolution multispectral imager (MRI), offering $22 \mathrm{~m}$ or $32 \mathrm{~m}$ GSD in up to four multiple spectral bands with a $300 \mathrm{~km}$ swath. Green red and near infra-red bands are equivalent to with LandSat Enhanced Thematic Mapper (ETM) bands $+2,+3$ and +4 respectively. The SLIM6 imager uses commercial lenses, linear array CCDs and electronics:
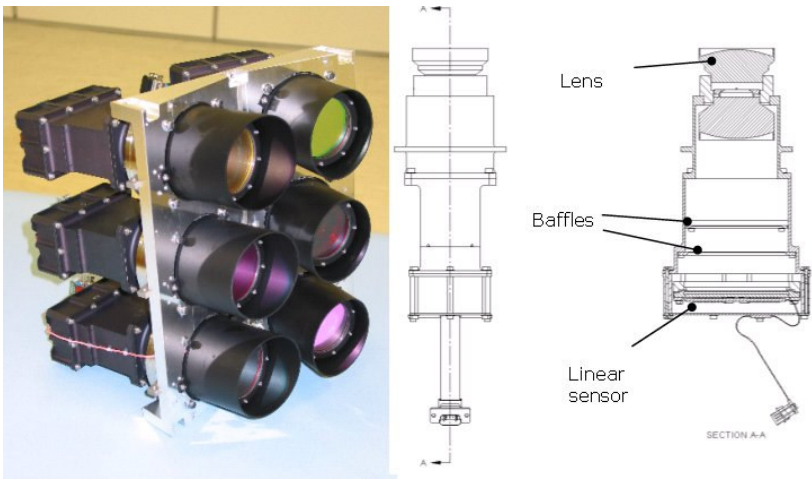

Figure 8. 32m GSD multispectral 'SLIM6' imager 
The 32m SLIM6 has already flown on five previous Disaster Monitoring Constellation (DMC) Missions. The improved 3-band, $22 \mathrm{~m}$ version will fly on two SSTL missions in 2008, and a 4-band (inc. blue color), 32m version will fly on NigeriaSat-2 in 2009.

\section{Other cameras}

A five band, medium resolution medium swath imager is also available, with performance lying between the VHRI and the SLIM6 multispectral imager. A similar camera will fly on the five RapidEye spacecraft due for launch in the second half of 2007. The CHRIS imager is a hyperspectral camera with resolution, imaged area and spectral resolution selectable remotely, the latter between 1.25 and $11 \mathrm{~nm}$. A CHRIS has been in operation on the PROBA spacecraft since 2001. The table below compares the primary imagers which SSTL spacecraft can carry, for a $686 \mathrm{~km}$ reference orbit altitude:

\begin{tabular}{|c|c|c|c|}
\hline Imager & $\begin{array}{c}\text { GSD at } \\
\text { nadir, (m) }\end{array}$ & $\begin{array}{c}\text { Swath or } \\
\text { tile (km) }\end{array}$ & Wavebands \\
\hline $\begin{array}{c}\text { VHR PAN } \\
\text { and M/S }\end{array}$ & $\begin{array}{c}\leq 2.5 \mathrm{PAN} \\
\leq 5 \mathrm{~m} \mathrm{M} / \mathrm{S}\end{array}$ & $20-30$ (tile) & $\begin{array}{c}\text { Up to 4: } \\
\text { NIR, R, G, B }\end{array}$ \\
\hline $\begin{array}{c}\text { Medium Res. } \\
\text { M/S }\end{array}$ & 7.1 & 91 & $\begin{array}{c}5, \text { across } \\
\text { VNIR }\end{array}$ \\
\hline $\begin{array}{c}\text { Wide Area } \\
\text { M/S }\end{array}$ & 22 & $300-600$ & $\begin{array}{c}\text { Up to 4: } \\
\text { NIR, R, G, B }\end{array}$ \\
\hline $\begin{array}{c}\text { CHRIS } \\
\text { hyperspectral }\end{array}$ & $\begin{array}{c}17 \mathrm{~m} \text { in best } \\
\text { mode }\end{array}$ & 13 (tile) & $\begin{array}{c}\text { Up to 62, 0.4- } \\
1.05 \mu m,\end{array}$ \\
\hline
\end{tabular}

TABLE I.

SSTL SPACECRAFT PAYLOAD PERFORMANCE COMPARISON

\section{Supporting subsystems}

SSTL-300 image data is compressed on-board using lossless data compression, for store-and-forward operations. Furthermore, switchable data encryption standard is available on the telemetry / telecommand, and switchable scrambling on payload data. Near real-time imaging \& down-linking is possible for a range of targets close to the ground station.

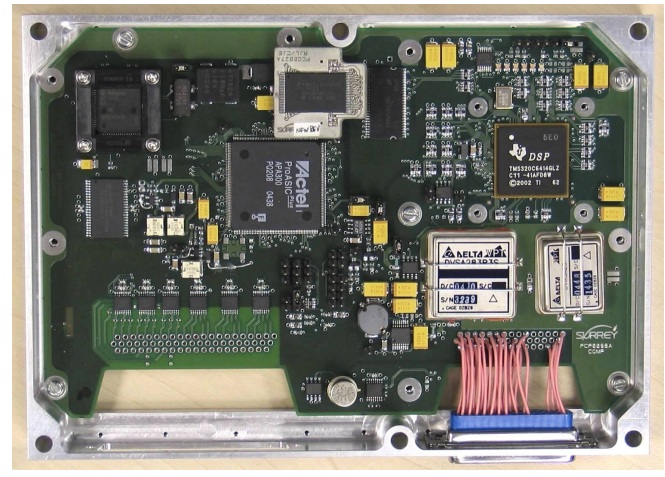

Figure 9. Beijing-1 digital signal processor (image compressor). Size is $\sim 160$ $\times 115 \mathrm{~mm}$, depth $30 \mathrm{~mm}$.

High performance geo-location (with and without use of ground control points) is provided, the fidelity exceeding $100 \mathrm{~m}$, dependent on the chosen subsystem options. On-board propulsion, not usually a feature of low cost microsatellites, is included for orbit maintenance, launcher injection correction, and (optionally) initial formation of a constellation.

\section{APPLICABLE IMAGING MODES}

The SSTL-300 is capable of simultaneous imaging using the VHRI and the MRI, and scenes can be as long as $2000 \mathrm{~km}$, as shown in Figure 10. A range of other imaging modes are available including: strip mapping, fast response scene capture, and stereo imaging to provide digital elevation models. A further high resolution area mode imaging mode stitches individual panchromatic or multispectral images together onboard the spacecraft to provide a tiled set of wide-swath and high-resolution imagery up to $60 \mathrm{~km}$ on a side.

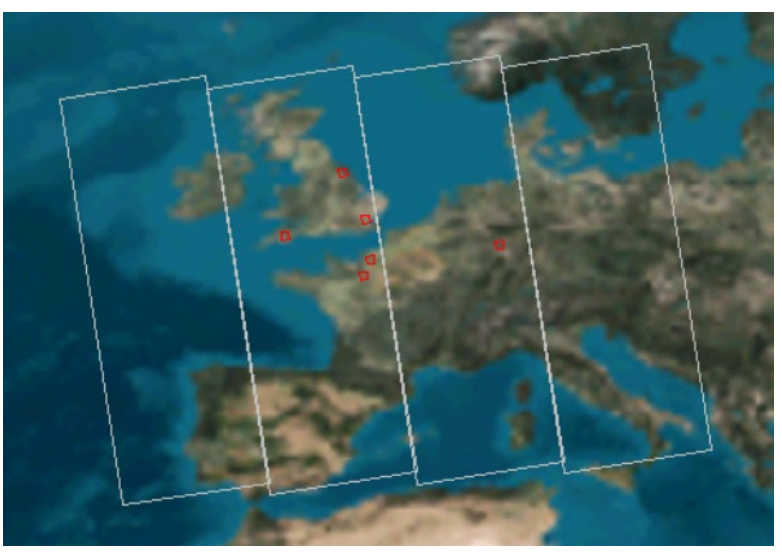

Figure 10. Combined wide swath and high resolution imaging modes

\section{MISSION PARAMETERS}

The nominal orbit for the SSTL-300 will be sunsynchronous, with a 10.30am node (time of equator crossing) to provide repeatable global coverage and good lighting conditions. The platform will orbit at approximately $700 \mathrm{~km}$, which provides good optimization for single satellite and constellation revisits, and allows compatibility of image data with the previous DMC spacecraft which orbit at an altitude of $686 \mathrm{~km}$.

SSTL through its low cost Earth observation platforms and constellations, pioneered by the DMC and continued with the NigeriaSat-2 SSTL-300 have introduced a new standard for generating cost effective Earth imagery from space.

\section{ACKNOWLEDGMENT}

SSTL would like to thank the National Space Research \& Development Agency (NASRDA) of Nigeria for funding the first flight of the SSTL-300, NigeriaSat-2, scheduled for 2009.

\section{REFERENCES}

[1] DigitalGlobe Constellation; Marketing brochure (Worldview 1). DigitalGlobe, Longmont CO, USA, April 2007. 